\title{
The Activation of the Mirror Neuron System during Action Observation and Action Execution with Mirror Visual Feedback in Stroke: A Systematic Review
}

\author{
Jack J. Q. Zhang, ${ }^{1}$ Kenneth N. K. Fong $\mathbb{D}^{1}{ }^{1}$ Nandana Welage, ${ }^{2}$ and Karen P. Y. Liu $\mathbb{D}^{2}$ \\ ${ }^{1}$ Department of Rehabilitation Sciences, The Hong Kong Polytechnic University, Kowloon, Hong Kong \\ ${ }^{2}$ School of Science and Health, Western Sydney University, Penrith, NSW, Australia \\ Correspondence should be addressed to Kenneth N. K. Fong; rsnkfong@polyu.edu.hk
}

Received 29 December 2017; Accepted 2 April 2018; Published 24 April 2018

Academic Editor: Ambra Bisio

Copyright @ 2018 Jack J. Q. Zhang et al. This is an open access article distributed under the Creative Commons Attribution License, which permits unrestricted use, distribution, and reproduction in any medium, provided the original work is properly cited.

\begin{abstract}
Objective. To evaluate the concurrent and training effects of action observation $(\mathrm{AO})$ and action execution with mirror visual feedback (MVF) on the activation of the mirror neuron system (MNS) and its relationship with the activation of the motor cortex in stroke individuals. Methods. A literature search using CINAHL, PubMed, PsycINFO, Medline, Web of Science, and SCOPUS to find relevant studies was performed. Results. A total of 19 articles were included. Two functional magnetic resonance imaging (fMRI) studies reported that MVF could activate the ipsilesional primary motor cortex as well as the MNS in stroke individuals, whereas two other fMRI studies found that the MNS was not activated by MVF in stroke individuals. Two clinical trials reported that long-term action execution with MVF induced a shift of activation toward the ipsilesional hemisphere. Five fMRI studies showed that AO activated the MNS, of which, three found the activation of movement-related areas. Five electroencephalography (EEG) studies demonstrated that AO or MVF enhanced mu suppression over the sensorimotor cortex. Conclusions. MVF may contribute to stroke recovery by revising the interhemispheric imbalance caused by stroke due to the activation of the MNS. AO may also promote motor relearning in stroke individuals by activating the MNS and motor cortex.
\end{abstract}

\section{Introduction}

Stroke is one of the leading causes of adult disability. Patients commonly suffer lasting motor impairments and functional disability following a stroke [1]. A substantial number of advanced rehabilitation strategies have been applied in upper limb stroke rehabilitation, such as robot-assisted therapy [2], constraint-induced movement training (CIMT) [3], and virtual reality- (VR-) based rehabilitation [4], which are aimed at helping stroke survivors relearn motor skills through intensive training. These rehabilitation strategies have been reported to improve patients' motor functions by inducing experience-dependent neuroplasticity in their damaged hemispheres [5-7]. However, the neuroplasticity resulting from intensive-based interventions may be limited if the residual motor functions of the patients are also extremely limited. It is crucial to find an adjunct therapy, on top of limb training, to enhance the recovery of the ipsilesional motor cortex of patients with severe hemiplegia, in order to overcome the learned nonuse phenomenon in such patient populations [8].

There is evidence to support the theory that cortical areas involved in motor execution can be activated by observing actions performed by others, which is attributed to the function of the mirror neuron system (MNS). The MNS is a class of neural substrates that discharges during action observation (AO) and action execution $[9,10]$. The MNS is also associated with various human functions, such as motor preparation [11], motion imitation [12, 13], language [14], and emotion recognition $[15,16]$. In humans, the core MNS is understood to be located in the inferior frontal gyrus (IFG), including the ventral premotor cortex (PMv), the inferior parietal lobule (IPL), and the intraparietal sulcus (IPS) $[9,17]$. An extended MNS involves additional brain regions, 
such as the primary motor cortex, the primary somatosensory cortex, and the middle frontal cortex [18]. A bilaterally distributed parietofrontal network with mirror neuron (MN) properties (i.e., parietofrontal MNS) has been proposed, which serves as a neural substrate to achieve the transformation of visual information into cortical areas for motor execution (i.e., visuomotor transformation) [19].

Based on this theory, researchers believe that the motor cortex could be primed by activating the MNS, thus boosting the efficacy of standardized rehabilitation for patients after strokes $[17,20]$. Subsequently, various rehabilitative strategies, aimed at facilitating the motor cortex through activating the MNS, have been applied in stroke rehabilitation, including action observation training (AOT) $[21,22]$ and action execution with MVF [23]. AOT usually consists of a session of $\mathrm{AO}$ followed by a session of imitating the observed action [22]. Some clinical trials have supported the efficacy of AO as a motor priming tool in stroke rehabilitation [24-28]. Previous neuroimaging studies have identified a bilateral AO network over the frontal, parietal, temporal, and occipital areas in the brain, which encompass the core MNS [29, 30]. Action execution with MVF, including mirror therapy (MT) [23], mirror box therapy [31], and VR-based MT [32], is already a commonly employed regimen in stroke rehabilitation. By virtue of MVF, patients receive a visual illusion showing that their hemiplegic upper limbs are moving normally when they move their nonparetic upper limbs simultaneously [23]. MVF could boost the effects of the conventional upper limb rehabilitation of stroke [33]. It has been proposed that the training-induced effects of MVF arise from the activation of the ipsilesional primary motor cortex by enriching the visual and proprioceptive inputs to the MNS [10, 19, 34], but this hypothesis has not been duly confirmed in human studies [35].

It is feasible nowadays for researchers to objectively measure the brain's activities before and after interventions using advanced functional neuroimaging and electrophysiological techniques [36]. An increasing number of studies regarding the effects of these two promising motor priming techniques ( $\mathrm{AO}$ and $\mathrm{MVF}$ ) on brain activation in stroke individuals have been published. However, there is a lack of focused reviews investigating the effects of $\mathrm{AO}$ or action execution with MVF on the activation of MNS and its subsequent effects on the activation of the motor cortex in patients who have had a stroke. We conducted this systematic review to evaluate the concurrent and training effects of $\mathrm{AO}$ and action execution with MVF on the activation of the MNS in stroke individuals, by reviewing available experimental studies as well as clinical trials with functional neuroimaging or electrophysiological examinations. In order to understand the role of the MNS in upper limb stroke rehabilitation, we have summarized the following information in this review: (1) MNS activation and (2) MNS activation and its relationship with the activation of the motor cortex.

\section{Methods}

2.1. Literature Search. A literature search for relevant studies was conducted using CINAHL (the Cumulative Index to
Nursing and Allied Health Literature), PubMed, PsycINFO, Medline, SCIE (Science Citation Index Expanded), and SCOPUS. Two of the authors of this review independently identified the relevant studies. Keywords used during the search were "stroke" OR "hemiplegia"; "action observation" OR "action observation training" OR "mirror visual feedback" OR "mirror neuron" OR "mirror therapy" OR “mirror box therapy"; and "functional imaging" OR "functional magnetic resonance imaging" OR "fMRI" OR "electroencephalography” OR "EEG” OR "near-infrared spectrometry” OR "NIRS” OR "magnetoencephalography" OR "MEG” OR "positron emission tomography" OR "PET". The date of publication was limited to 10 years, from January 2007 to November 2017. The reference lists of the retrieved articles were manually searched to identify any further relevant articles.

2.2. Selection Criteria. We used the PICOS method to formulate our selection criteria. Studies that satisfied all the following criteria were considered for this review.

Population $(P)$ : studies recruiting adult patients diagnosed with having had strokes; intervention (I): interventions or experimental paradigms using $\mathrm{AO}$ or $\mathrm{MVF}$ in regard to upper limb actions; comparison (C): control conditions without AO or MVF or using sham AO or MVF; outcomes $(O)$ : studies providing anatomical evidence of brain activation induced by $\mathrm{AO}$ or action execution with MVF, as represented by signal changes in PET, fMRI, or fNIRS; or using previously validated electrophysiological indices of MN activities, such as event-related desynchronization (ERD) of the mu band (i.e., mu suppression) [37] or the ERD of the beta band (i.e., beta suppression) [38]; or employing an advance analysis to explore the neural network related to AO or MVF, including but not limited to dynamic casual modelling (DCM) in regard to fMRI or a coherence analysis of EEG; and study design (S): clinical trials investigating the training effects or experimental studies investigating the concurrent effects of the relevant experimental condition.

Studies were excluded if (1) they only recruited healthy subjects or patients with other primary diagnoses (e.g., Parkinson's disease); (2) they only focused on the lower limb or trunk actions; (3) the final analyzed sample size was less than five; (4) they were published as conference proceedings, dissertations, or in books; and (5) they were not published in English language.

2.3. Data Extraction. After identifying relevant studies, two authors independently extracted the following information from each article: (1) the characteristics of participants; (2) the protocol of the intervention or experiment; (3) the modalities of the functional neuroimaging or electrophysiological techniques used in the study; and (4) the main results of the studies. Any disagreement was settled by discussion with the third author.

2.4. Quality Assessment. We assessed the quality of the randomized controlled trials (RCTs) in regard to the training effects of AOT or action execution with MVF in patients who have had strokes, based on the Physiotherapy Evidence Database (PEDro) scale. Both independent reviewers evaluated 
each article. The PEDro scale consists of 11 items. The first criterion, item eligibility, is not scored, as it is used as a component of external validity. The other criteria included random allocation, concealment of allocation, baseline equivalence, blinding procedure, intention to treat analysis, adequate follow-up, between-group statistical analysis, measurement of data variability, and point estimates. Any scoring discrepancies were resolved.

\section{Results}

3.1. Identification Process for the Selection of the Studies. The initial search yielded 332 results. After removing duplicates, a total of 191 records were screened, of which, 138 citations were excluded for the following reasons: the studies were reviews or meta-analyses $(n=18)$; the studies' protocols $(n=6)$; the studies focused on infants, children, or adolescents $(n=4)$; the studies enrolled only healthy participants or patients with neurological diseases other than strokes $(n=58)$; or the studies were irrelevant $(n=52)$. The remaining 53 articles were subjected to full-text reading, of which 34 articles were removed for the following reasons: the studies did not use functional neuroimaging or electrophysiological techniques for stroke participants $(n=17)$; the final analyzed sample sizes of the studies were less than five $(n=8)$; studies of motion observation with a brain-computer interface $(n=2)$; the visual feedback was based on the lower limbs or trunk actions, rather than upper limb actions $(n=4)$ [39-42]; or the studies used EEG spectrum analysis alone $(n=1)$ [43]. One study focused on the functional neuroplasticity induced by observing the skills of tool use, which was hard to compare with other protocols of AO. Another study enrolled both patients who have had strokes and brain tumors, and the data of stroke participants could not be separated; these studies were hence excluded $[16,44]$. Finally, 19 articles satisfied our inclusion criteria and were included in the present review $[13,22,28,34$, 45-59]. Figure 1 shows the identification process for the selection of studies.

3.2. Clinical Trials regarding the Training Effects of LongTerm Intervention. Among the included studies, six studies focused on the training effects of long-term therapeutic programs $[22,28,45,46,52,59]$. Four of these were RCTs $[22,28,45,52]$, and the other two were interventional studies with pre-post comparisons $[46,59]$. Three studies investigated the training effects of bimanual training with MVF (four- to eight-week interventions) $[45,46,52]$. Of these, two identified a shift of activation toward the ipsilesional hemisphere $[46,52]$, evidenced by fMRI. Cortical areas activated by MVF mainly included the primary motor cortex $[46,52]$ and the premotor cortex (PMC) [46]. A study with EEG reported that mu suppression over sensorimotor cortex (SMC) was higher in the group with MVF [45]. Sun et al., who also used mu suppression as an index, reported additional benefits of $\mathrm{AO}$ on the basis of motor imagery (MI) in regard to enhancing $\mathrm{mu}$ suppression over the ipsilesional SMC, compared with the control group (MI alone) [28]. A fNIRS study measured the difference in brain activity of participants before and after four weeks of MT in addition to conventional rehabilitation; however, the difference in the activation pattern over the primary motor cortex and the precuneus was insignificant over time [59].

An RCT investigating the training effects of $\mathrm{AO}$ found that the four-week AOT ( $\mathrm{AO}$ followed by imitation) induced more evident activation over bilateral PMv, bilateral superior temporal gyrus (STG), supplementary motor area (SMA) over the contralesional hemisphere, and supramarginal gyrus (SMG) over the ipsilesional hemisphere, relative to the control group watching nonbiological videos followed by action execution [22]. Characteristics of these studies are summarized in Table 1.

3.3. Experimental Studies with fMRI Findings. Eight articles explored the concurrent effects of $\mathrm{AO}$ or action execution with MVF on brain activation, evidenced by fMRI [34, 47, $48,50,53,55-57]$. In studies regarding MVF, Michielsen et al. found that bimanual movement with MVF led to significant activation of the precuneus and the posterior cingulate cortex (PCC), rather than the MNS [53]. However, Saleh et al. $[34,55]$ reported that the ipsilesional primary motor cortex was activated by MVF, and connectivity between the ipsilesional primary somatosensory cortex and the primary motor cortex was stronger, relative to the control group without MVF [55]. The source of the ipsilesional primary motor cortex activation was further found by the DCM to be the contralesional intraparietal sulcus (IPS) [34]. Wang et al. reported that lateralized activation toward the affected hemisphere was in favor of virtual MVF, as reflected by the peak $T$ value of the precuneus in the majority of their samples [57].

For studies concerning AO, Szameitat et al. found that $\mathrm{AO}$ of wrist movement activated the PMC and IPL; however, the pattern of neural activation in action execution more resembled MI, rather than AO [56]. Garrison et al. reported that left IFG, SMG, and bilateral precentral gyrus were activated during right-hand (paretic side) observation of reach and grasp actions. Lateralized activation toward the ipsilesional hemisphere was also noted [50]. During left-hand $\mathrm{AO}$ (nonparetic side), the bilateral activation was relatively symmetrical in stroke individuals. Brunner et al. [47] devised a protocol to observe bimanual action; they reported that stroke individuals (within one-to-two weeks after the stroke) showed activation in inferior and superior parietal lobes, IFG, and the primary motor cortex during $\mathrm{AO}$. In the second fMRI exam (three months after the stroke), the neural response to $\mathrm{AO}$ was extended to more movement-related areas, including the PMC, primary motor cortex, and the SMA. The neural response to $\mathrm{AO}$ was increased from one or two weeks to three months after the individual suffered a stroke. Dettmers et al. compared the brain activities during $\mathrm{AO}$ and MI of patients with left or right subcortical strokes and reported that patients with left subcortical strokes presented higher levels of activity than those with right subcortical strokes [48]. The brain activation induced by $\mathrm{AO}$ or MVF shown by fMRI are summarized in Table 2.

3.4. Experimental Studies with EEG or MEG. Four experiments measured the concurrent effects of AO on mu rhythm 

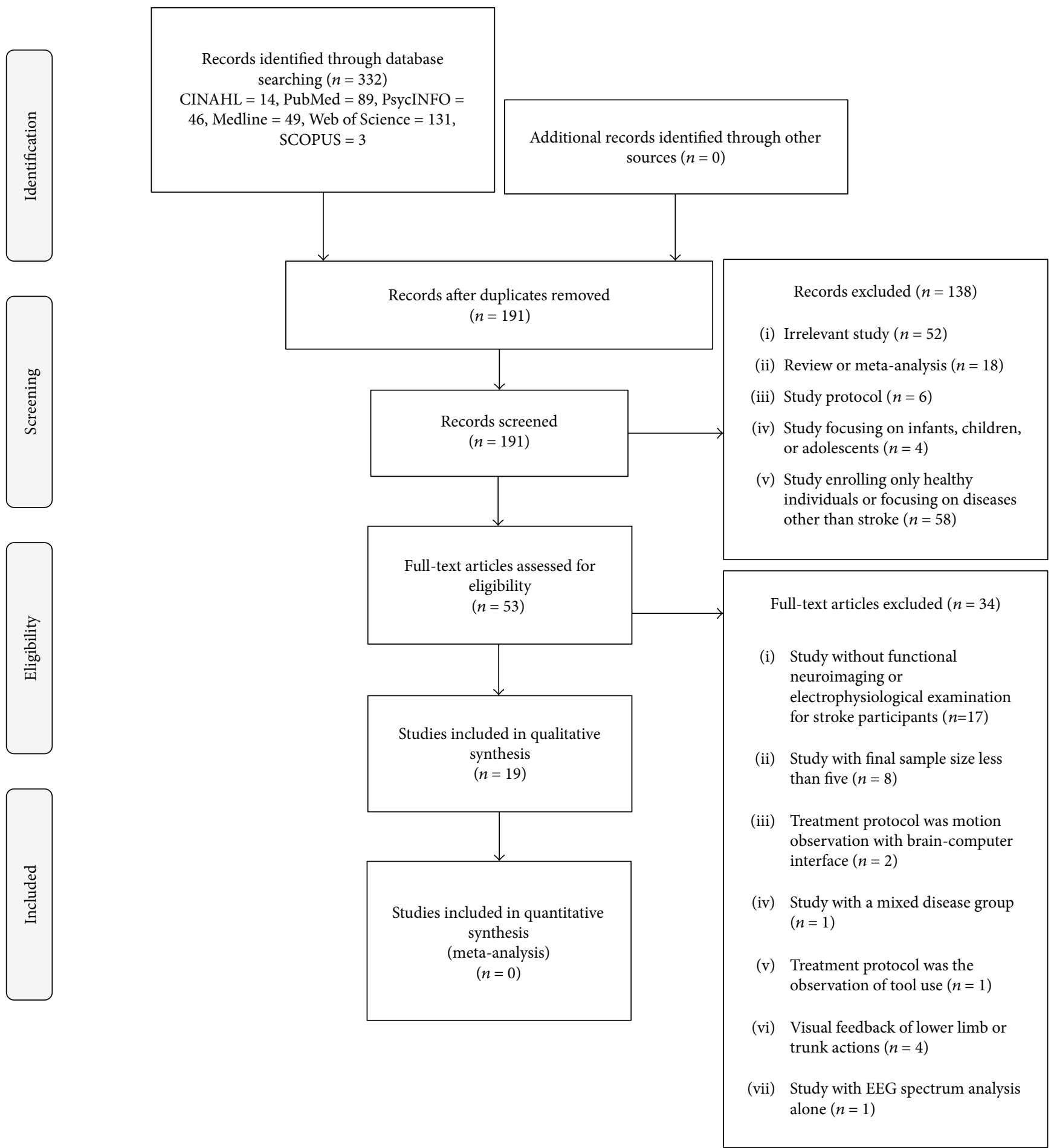

Figure 1: Flowchart of literature search.

using EEG [13, 49, 51, 58]. Another study used MEG to measure the difference in beta suppression during bimanual movement with and without MVF [54]. Kuk et al. reported the whole brain topography based on mu rhythm before and after $\mathrm{AO}$ and found that the middle frontal gyrus (MFG) was less active after a total of five sessions of $\mathrm{AO}$ [51]. Frenkel-Toledo et al. reported that observations of the reach-and-grasp hand action could induce mu suppression over the SMC, but the magnitude of mu suppression was significantly lower in the affected hemisphere, relative to the unaffected hemisphere. $\mathrm{Mu}$ suppression over the unaffected side was attenuated in patients with lesions over the right IPL $[13,49]$. Tani et al. also showed that AO of the open-and-close action by the paretic hand induced stronger 


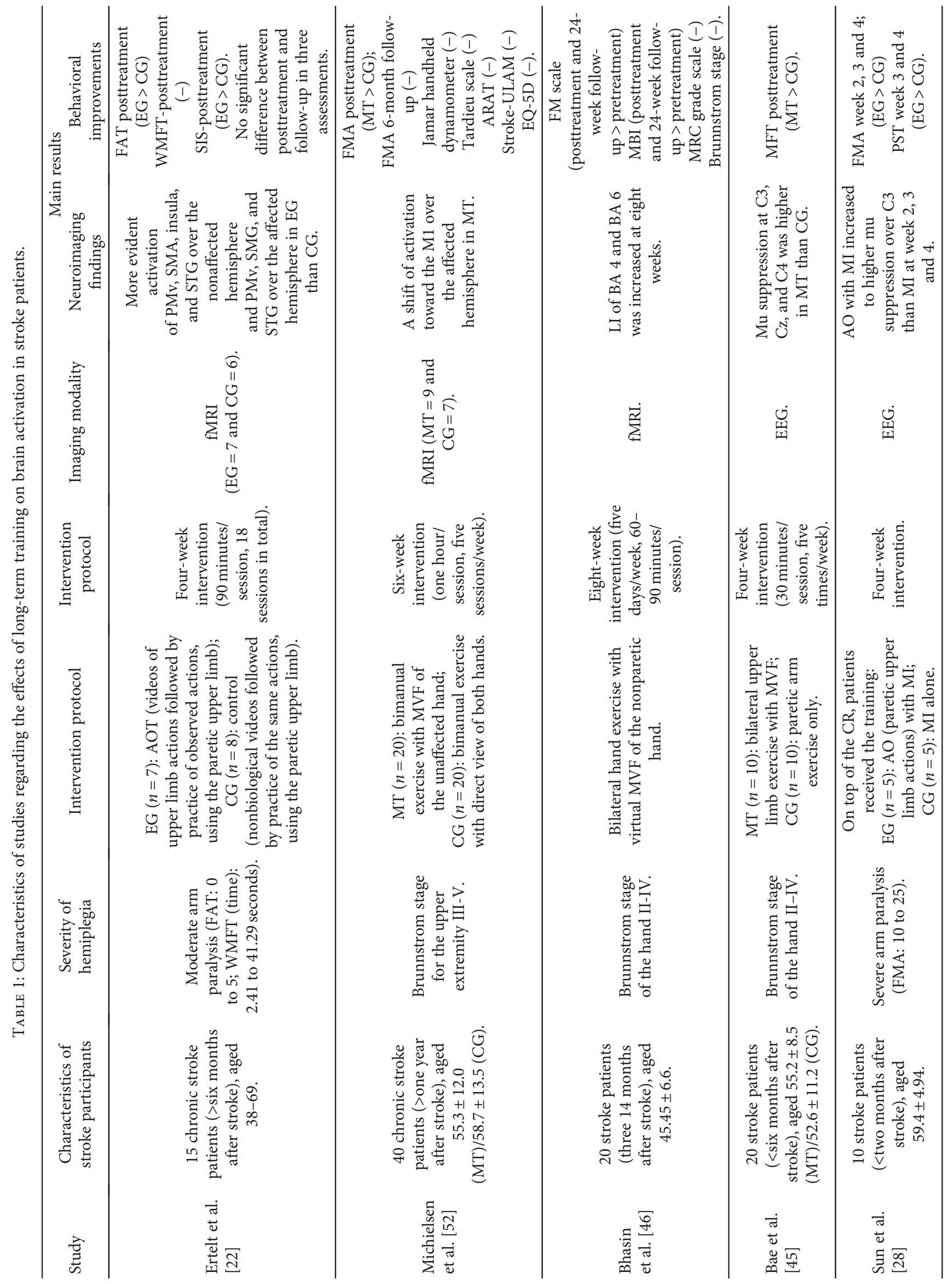




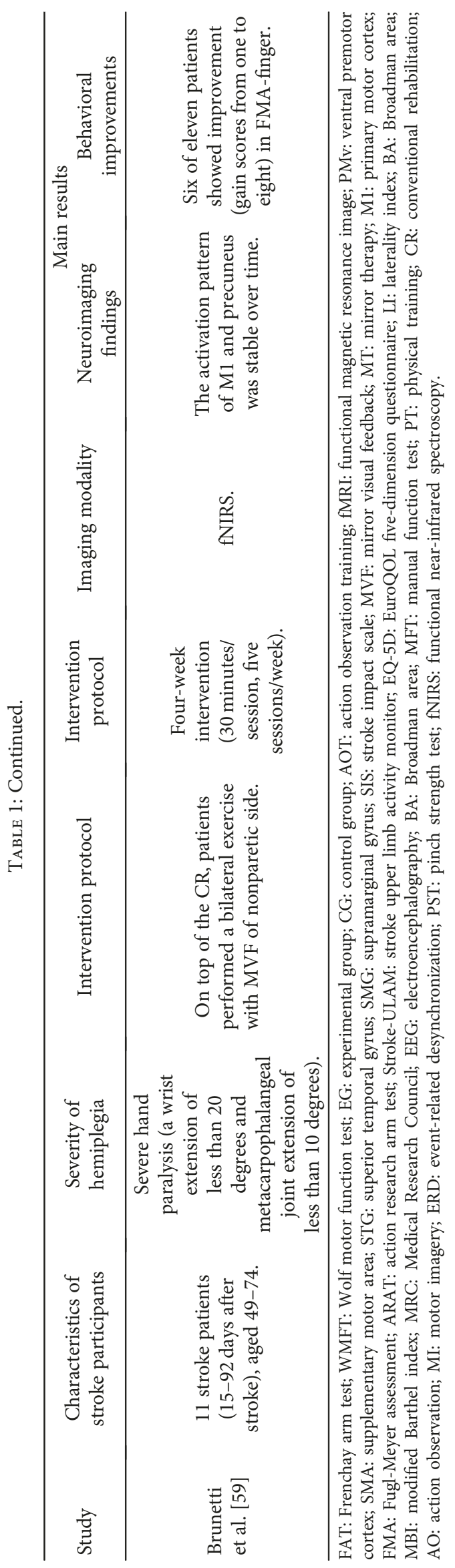


TABLE 2: Brain activation or lateralization of brain activation measured by fMRI.

\begin{tabular}{|c|c|c|c|c|}
\hline Brain areas & $\begin{array}{c}\text { Concurrent effect of } \\
\text { MVF versus the control } \\
\text { without MVF during a } \\
\text { motor task }\end{array}$ & $\begin{array}{l}\text { Long-term rehabilitation } \\
\text { with MVF versus the control } \\
\text { without MVF }\end{array}$ & $\begin{array}{l}\text { Concurrent effect of AO } \\
\text { versus the control }\end{array}$ & $\begin{array}{c}\text { Long-term rehabilitation } \\
\text { with } \mathrm{AO} \text { versus the control } \\
\text { without } \mathrm{AO}\end{array}$ \\
\hline \multicolumn{5}{|l|}{ Frontal lobes } \\
\hline $\begin{array}{l}\text { Primary motor } \\
\text { cortex }\end{array}$ & $\begin{array}{c}\text { Ipsilesional } \\
\text { activation }[34,55]\end{array}$ & $\begin{array}{c}\text { Ipsilesional lateralization } \\
{[46,52]}\end{array}$ & $\begin{array}{c}\text { Bilateral activation [47]; } \\
\text { ipsilesional lateralization [50] }\end{array}$ & \\
\hline Premotor cortex & & Ipsilesional lateralization [46] & $\begin{array}{c}\text { Bilateral activation [47]; } \\
\text { ipsilesional activation [56] }\end{array}$ & Bilateral activation [22] \\
\hline $\begin{array}{l}\text { Supplementary } \\
\text { motor area }\end{array}$ & & & Bilateral activation [47] & $\begin{array}{c}\text { Contralesional activation } \\
{[22]}\end{array}$ \\
\hline $\begin{array}{l}\text { Superior frontal } \\
\text { gyrus }\end{array}$ & & & Ipsilesional activation [48] & \\
\hline $\begin{array}{l}\text { Inferior frontal } \\
\text { gyrus }\end{array}$ & & & $\begin{array}{c}\text { Bilateral activation [47]; } \\
\text { ipsilesional lateralization } \\
{[48,50]}\end{array}$ & \\
\hline Prefrontal gyrus & & & Ipsilesional activation [48] & \\
\hline \multicolumn{5}{|l|}{ Parietal lobes } \\
\hline $\begin{array}{l}\text { Primary } \\
\text { somatosensory } \\
\text { cortex }\end{array}$ & $\begin{array}{l}\text { Bilateral activation } \\
{[34,55]}\end{array}$ & & & \\
\hline $\begin{array}{l}\text { Superior parietal } \\
\text { gyrus }\end{array}$ & & & $\begin{array}{l}\text { Bilateral activation [47]; } \\
\text { ipsilesional activation [48] }\end{array}$ & \\
\hline Precuneus & $\begin{array}{c}\text { Bilateral activation } \\
{[34,53,55] \text {; ipsilesional }} \\
\text { lateralization [57] }\end{array}$ & & & \\
\hline $\begin{array}{l}\text { Inferior parietal } \\
\text { gyrus }\end{array}$ & & & $\begin{array}{l}\text { Ipsilesional activation [56]; } \\
\text { bilateral activation [47] }\end{array}$ & \\
\hline $\begin{array}{l}\text { Supramarginal } \\
\text { gyrus }\end{array}$ & $\begin{array}{c}\text { Contralesional } \\
\text { activation }[34,55]\end{array}$ & & Ipsilesional lateralization [50] & Ipsilesional activation [22] \\
\hline $\begin{array}{l}\text { Intraparietal } \\
\text { sulcus }\end{array}$ & $\begin{array}{c}\text { Contralesional } \\
\text { activation }[34,55]\end{array}$ & & & \\
\hline $\begin{array}{l}\text { Posterior cingular } \\
\text { cortex }\end{array}$ & $\begin{array}{l}\text { Contralesional } \\
\text { activation [53] }\end{array}$ & & & \\
\hline
\end{tabular}

Temporal lobes

Superior

temporal gyrus

Bilateral activation [22]

Inferior temporal

gyrus

Bilateral activation [47]

Occipital lobes

Occipital gyrus

Bilateral activation [47]

Notes: MVF: mirror visual feedback; AO: action observation. Garrison et al. [50]: results of AO of the paretic hand movement were used; Bhasin et al [46]: the result of within-group difference was used, because the study did not have a control group.

mu suppression than the MI of the same action in stroke individuals [58]. Interhemispheric imbalance of movementrelated beta suppression was noted in stroke participants in the study by Rossiter et al. when performing the bimanual open-and-close action. The initial asymmetricity was partially attenuated by MVF [54]. Characteristics of the experimental studies are summarized in Table 3 , and brain activation induced by $\mathrm{AO}$ or MVF, measured by fMRI, is summarized in Table 2.

3.5. Methodological Quality of Included Randomized Controlled Trials. Four RCTs were included in this review
$[22,28,45,52]$. The results of the assessment of methodological quality are summarized in Table 4.

\section{Discussion}

The present study is aimed at systematically evaluating the evidence of MNS activation induced by AO or MVF and its potential effects on the activation of the motor cortex in patients who have had strokes. The main findings of the present review are (1) the ipsilesional primary motor cortex can be facilitated by MVF $[46,52]$, which may be achieved by recruiting the MNS $[34,55]$; (2) long-term action execution 


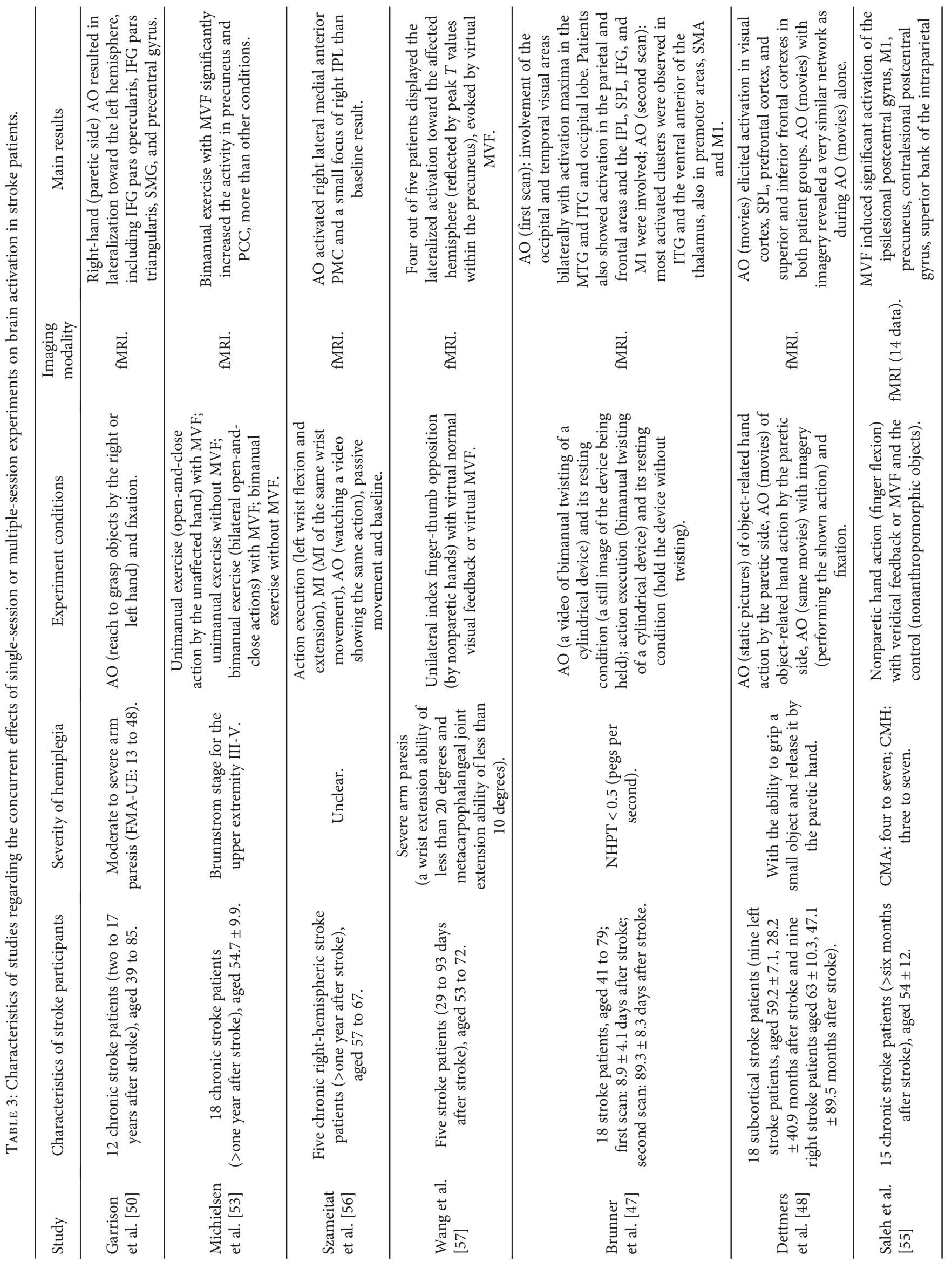




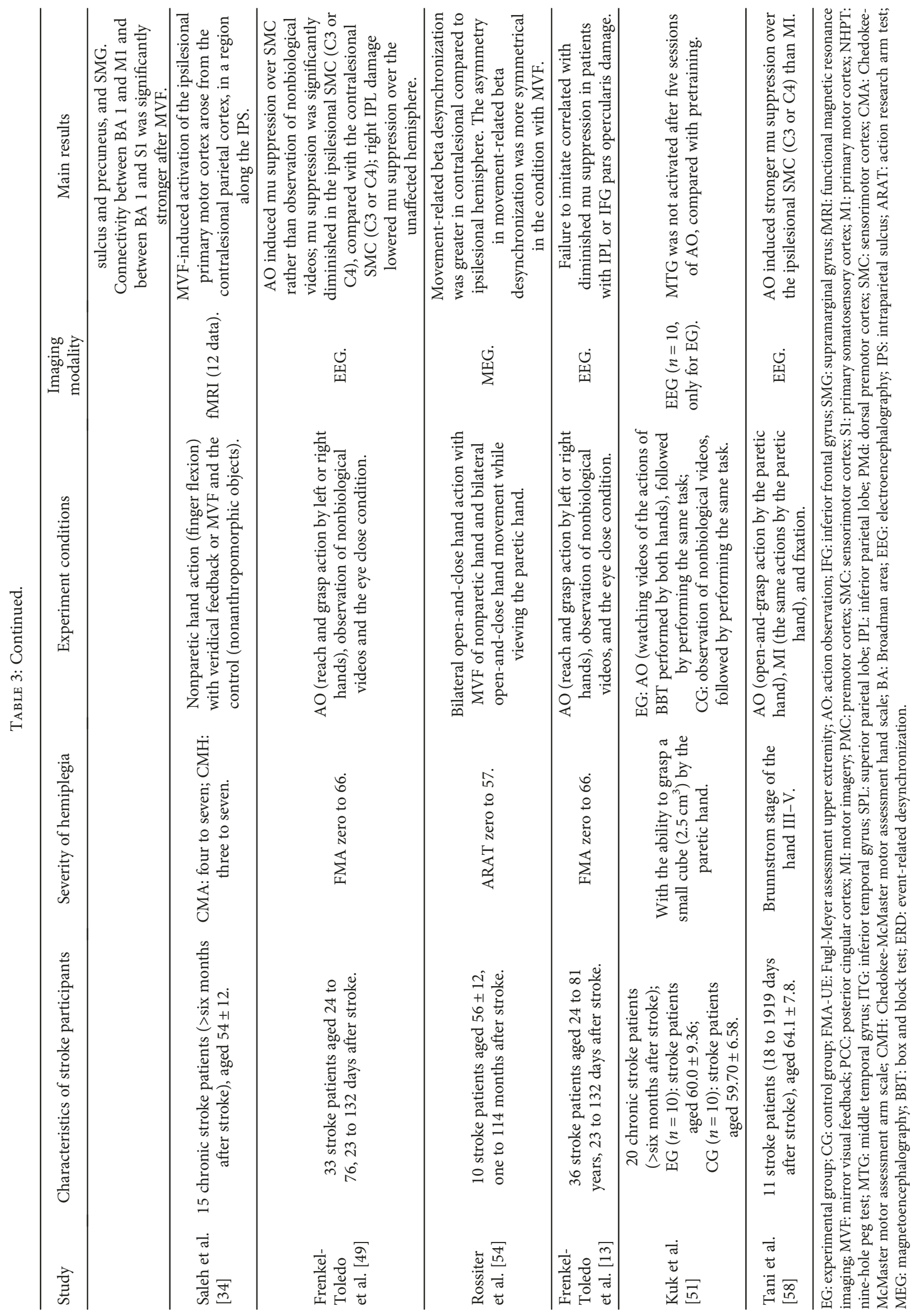


TABLE 4: Methodological assessment of included studies using the PEDro scale*.

\begin{tabular}{|c|c|c|c|c|}
\hline Criterion & $\begin{array}{l}\text { Ertelt } \\
\text { et al. [22] }\end{array}$ & $\begin{array}{l}\text { Michielsen } \\
\text { et al. [52] }\end{array}$ & $\begin{array}{c}\text { Bae } \\
\text { et al. [45] }\end{array}$ & $\begin{array}{l}\text { Sun } \\
\text { et al. [28] }\end{array}$ \\
\hline Eligibility criteria & Yes & Yes & No & Yes \\
\hline $\begin{array}{l}\text { Random } \\
\text { allocation }\end{array}$ & 1 & 1 & 1 & 1 \\
\hline $\begin{array}{l}\text { Concealed } \\
\text { allocation }\end{array}$ & 0 & 1 & 0 & 0 \\
\hline $\begin{array}{l}\text { Baseline } \\
\text { comparability }\end{array}$ & 1 & 1 & 1 & 1 \\
\hline Blind subjects & 0 & 0 & 0 & 0 \\
\hline Blind therapists & 0 & 0 & 0 & 0 \\
\hline Blind assessors & 0 & 1 & 0 & 1 \\
\hline $\begin{array}{l}\text { Adequate } \\
\text { follow-up }\end{array}$ & 1 & 1 & 0 & 1 \\
\hline $\begin{array}{l}\text { Intention-to- } \\
\text { treat analysis }\end{array}$ & 0 & 1 & 0 & 1 \\
\hline $\begin{array}{l}\text { Between group } \\
\text { comparisons }\end{array}$ & 1 & 1 & 1 & 1 \\
\hline $\begin{array}{l}\text { Point estimates } \\
\text { and variability }\end{array}$ & 1 & 1 & 1 & 1 \\
\hline Total scores & 5 & 8 & 4 & 7 \\
\hline
\end{tabular}

*The PEDro scores were taken from the PEDro website, except Ertelt et al. [22] and Sun et al. [28], which were rated by our team.

with MVF resulted in a shifted activation toward the ipsilesional hemispheres in patients who have had strokes; hence, a more symmetrical state between the two hemispheres may be achieved $[46,52]$; (3) AO induced broader brain activation in the frontal, parietal, temporal, and occipital areas in patients who have had strokes, which encompassed the MNS, as well as cortical areas of motor execution, including the primary motor cortex, PMC, and SMA [22, 47, 48, 50]; (4) mu suppression can be induced by $\mathrm{AO}$ in patients who have had strokes; however, mu suppression over the affected hemisphere is relatively diminished $[13,49,58]$; and (5) MVF [45] or AO [28] embedded in long-term rehabilitation could bring about additional neurophysiological effects in patients after they have had strokes, reflected by more evident mu suppression, which may indicate that $\mathrm{MN}$ activities can be increased by this training.

A classical pathological change following stroke involves the activities of the affected hemispheres being suppressed, while those of less affected hemispheres are heightened, due to interhemispheric competition rivalry [60]; hence, successful motor recovery in patients who have had strokes could be achieved by normalizing the interhemispheric asymmetricity and promoting the neuroplasticity of the ipsilesional motor cortex $[61,62]$. As the present review has shown, long-term MVF can contribute to a shift in activation toward the affected hemisphere [46, 52]. Furthermore, MVF transiently attenuates the asymmetric activities of movement-related beta suppression [54]. These findings can partially explain the beneficial effects of MT, which induces more symmetrical activities between the two hemispheres in patients who have had strokes. This is in line with previous findings regarding the effects of MVF on the healthy brain [35]. However, evidence to support the way in which $\mathrm{AO}$ can induce a shift of activation toward the affected hemisphere is relatively limited [50].

The difference between the activation patterns of MVF and $\mathrm{AO}$ can be identified. MVF mainly activates the ipsilesional primary motor cortex $[34,40,46,52,55]$, the PMC [46], the primary somatosensory cortex $[34,40,55]$, and the IPL $[34,55]$. Two articles (one study) using effective connectivity and DCM have proposed that MVF could increase the connectivity between the ipsilesional primary somatosensory and primary motor cortex [55]. This study also suggests that the activation of the ipsilesional primary motor cortex may arise from contralesional IPS [34], which is a part of the MNS. These results are in line with the assumed functions of the MNS: visuomotor transformation. However, this conclusion should be interpreted with caution, since some studies have not identified the MNS activation by MVF in either patients who have had strokes [53] or healthy subjects [35]. The studies by Saleh et al. did not choose another frontal MNS (e.g., PMC) as the node in the DCM [34, 55]. As the first fMRI study with DCM that supports the activation of the MNS induced by MVF and its subsequent effects on the activation of the primary motor cortex [34], it is worthwhile to further explore the neural network underlying the MVF, in order to explain the role of the MNS in the network.

For AO, the activated brain regions were much broader, including IFG [47, 50], the PMC [22, 47, 48], the IPL [47], the primary motor cortex $[47,50]$, and temporal and occipital structures, which encompass the parietofrontal MNS as well as the cortical areas for motor execution (e.g., the primary motor cortex, PMC, and SMA). Small et al. proposed a model of brain repair after a stroke, which hypothesized that the MNS activation induced by AO may promote the reorganization of the cortical motor loop (i.e., the primary motor cortex, PMC, and SMA), thereby improving the motor functions of stroke survivors [63]. Our findings provide anatomical evidence to support this model. Observations of the bimanual action elicited a similar activation pattern as the execution of the same action in one study [47], whereas another study showed that $\mathrm{AO}$ (of a simple wrist movement) activated a part of the PMC and IPL, which did not resemble the activation pattern of action execution to such a great extent [56]. This difference may be attributed to different experimental paradigms of $\mathrm{AO}$. The $\mathrm{AO}$ network, which may be involved in the understanding of motor intention, may have a stronger response to an object-directed or goaldirected action $[64,65]$, relative to a single action without meaning, although this is still inconclusive [66]. All in all, the results are still consistent with previously defined bilateral AO networks in healthy human brains $[29,30]$. The neural network underlying the $\mathrm{AO}$ remains unclear.

The activation patterns of MVF and AO are obviously different. AO elicited broader activation of the frontal, parietal, temporal, and occipital areas, while the activated regions of MVF mainly covered the frontal and parietal structures. The difference between these two regimens is that the participants were required to perform bilateral or unilateral movement themselves when observing the visual feedback in the 
MVF experiments, while this was not required in the AO experiments. Previous studies have assumed that there are different neural networks in response to $\mathrm{AO}$ and MVF $[35,67]$, and this opinion was confirmed by the present review in the stroke cohorts. Even though the underlying neural network cannot be fully understood at this stage, MNS activation seems to play a key role in both AO- and MVFinduced functional plasticities. There have been several TMS studies that have provided indirect evidence of the activation of the primary motor cortex by AO or MVF $[27,68]$. Therefore, these two modalities can be viewed as optional motor priming tools for stroke rehabilitation. However, there is still a lack of studies directly comparing the clinical improvements and the pattern of neuroplasticity induced by MVF and AO [69] and whether or not the activation pattern is congruent with the clinical improvements in patients with stroke.

Brain waveforms recorded by EEG are altered by AO, reflected by lower alpha power and higher beta power over the frontal, central, and occipital electrodes [41, 42]. Some studies have also shown that the electrophysiological responses to $\mathrm{AO}$ may decrease after repetitive stimulations $[43,51]$. These changes may be related to the changes in cognitive activities related to the understanding of motor intention after receiving visual feedback [70]. However, pure spectrum analysis is less likely than detailed EEG analyses, for example, time-frequency analysis, to reflect $\mathrm{MN}$ activity and stroke recovery $[71,72]$. A recent meta-analysis indicated that both $\mathrm{AO}$ and action execution could induce the suppression of mu rhythm with a significant effect size [73]. This property of dual activation makes the mu suppression a signature of human mirror neuron activity [37, 74]. Regarding the studies included in the present review, two articles investigated $\mathrm{AO}$-induced mu suppression in stroke individuals and its relationship with brain lesions [13, 49]. They found that the magnitude of mu suppression was reduced in the affected hemisphere, relative to the unaffected hemisphere, which was also identified by another study [58]. Two RCTs demonstrated that AO- or MI-induced mu suppression can be enhanced after long-term AOT [28] or training with MVF [45], which implies enhanced MN activities after the $\mathrm{AO}$ or MI training. Lesion analysis also showed that the damage over IPL or IFG was correlated with the diminished mu suppression, which indicates that mu suppression may be a specific index of MN activities [13, 16, 49].

One study demonstrated that behavioral improvement is correlated with the neural response to $\mathrm{AO}$ (measured by fMRI) in stroke individuals, which indicates that the AOinduced neural response is likely to be an indicator that can evaluate the arm motor recovery of patients who have had strokes within the first three months [47]. As the MNS is correlated with motion imitation [75], its activation may be a neurobiomarker that can measure the potential of motor learning in patients who have had strokes [47], which could also be measured by mu suppression. The predictive value of mu suppression and its relationship with motor improvement remain speculative. Other frequency bands related to action execution and AO, such as the beta band, might also be suitable candidates in regard to measuring $\mathrm{MN}$ activities $[38,76]$, although evidence of this in the stroke population is fairly limited [54]. As EEG is a relatively low-cost technique, further studies are encouraged to longitudinally explore the role of the ERD of different frequency ranges over various brain regions in patients who have had strokes. This neurobiomarker may also serve as a useful reference of the patients' motor recovery trajectory and motor relearning potential, promoted by MNS activation in stroke individuals.

There are some limitations in the present review. First, the heterogeneous protocols of $\mathrm{AO}$ and MVF and different experimental designs in regard to implementing neuroimaging hindered us from giving a firm and precise conclusion. Second, potentially confounding factors cannot be fully explained, based on currently available evidence, such as the dominance of handedness [48] and the nature of stroke lesions [48], which may result in different responses to AO and MVF. Further studies are warranted to answer these questions. Finally, restricting our review to English publications may have resulted in language bias.

\section{Conclusions}

MVF may contribute to stroke recovery by revising the interhemispheric imbalance, and MNS recruitment may be one of the potential neural mechanisms in this process. $\mathrm{AO}$ is associated with the activation of the MNS and motor cortex, which may promote motor relearning in stroke individuals. More rigorous studies with functional neuroimaging or electrophysiological techniques should be performed to further explain the different functional neural networks underlying $\mathrm{AO}$ or MVF and to explore the relationship between MN activities and clinical recovery in patients who have had strokes.

\section{Abbreviations}

AO: Action observation

MVF: Mirror visual feedback

MNS: Mirror neuron system

fMRI: Functional magnetic resonance imaging

EEG: Electroencephalography

CIMT: Constraint-induced movement training

VR: Virtual reality

IFG: Inferior frontal gyrus

PMv: Ventral premotor cortex

IPL: Inferior parietal lobule

IPS: Intraparietal sulcus

MT: Mirror therapy

MEG: Magnetoencephalography

PET: Positron emission tomography

NIRS: Near-infrared spectrometry

ERD: Event-related desynchronization

DCM: Dynamic casual modelling

PMC: Premotor cortex

SMC: Sensorimotor cortex

MI: Motor imagery

STG: Superior temporal gyrus

SMA: Supplementary motor area

SMG: Supramarginal gyrus

PCC: Posterior cingulate cortex

MFG: Middle frontal gyrus. 


\section{Conflicts of Interest}

The authors declare that they have no conflicts of interest.

\section{Acknowledgments}

This work was partially funded by a grant from the General Research Fund (GRF) (Grant no. 151039/15M), Research Grants Council, University Grants Committee, Hong Kong, to Kenneth N. K. Fong.

\section{References}

[1] B. H. Dobkin, "Rehabilitation after stroke," New England Journal of Medicine, vol. 352, no. 16, pp. 1677-1684, 2005.

[2] A. C. Lo, P. D. Guarino, L. G. Richards et al., "Robot-assisted therapy for long-term upper-limb impairment after stroke," New England Journal of Medicine, vol. 362, no. 19, pp. 1772$1783,2010$.

[3] G. Kwakkel, J. M. Veerbeek, E. E. H. van Wegen, and S. L. Wolf, "Constraint-induced movement therapy after stroke," The Lancet Neurology, vol. 14, no. 2, pp. 224-234, 2015.

[4] K. E. Laver, S. George, S. Thomas, J. E. Deutsch, and M. Crotty, "Virtual reality for stroke rehabilitation," Cochrane Database of Systematic Reviews, no. 2, article CD008349, 2015.

[5] Y. T. Fan, K. C. Lin, H. L. Liu, C. Y. Wu, Y. Y. Wai, and T. H. Lee, "Neural correlates of motor recovery after robot-assisted stroke rehabilitation: a case series study," Neurocase, vol. 22, no. 5, pp. 416-425, 2016.

[6] J. Liepert, W. H. R. Miltner, H. Bauder et al., "Motor cortex plasticity during constraint-induced movement therapy in stroke patients," Neuroscience Letters, vol. 250, no. 1, pp. 5-8, 1998.

[7] S. Saleh, G. Fluet, Q. Qiu, A. Merians, S. V. Adamovich, and E. Tunik, "Neural patterns of reorganization after intensive robot-assisted virtual reality therapy and repetitive task practice in patients with chronic stroke," Frontiers in Neurology, vol. 8, no. 452, p. 452, 2017.

[8] V. Ramachandran, "Plasticity and functional recovery in neurology," Clinical Medicine, vol. 5, no. 4, pp. 368-373, 2005.

[9] G. Rizzolatti and L. Craighero, "The mirror-neuron system," Annual Review of Neuroscience, vol. 27, no. 1, pp. 169-192, 2004.

[10] G. Rizzolatti and G. Luppino, "The cortical motor system," Neuron, vol. 31, no. 6, pp. 889-901, 2001.

[11] L. Fadiga, L. Fogassi, G. Pavesi, and G. Rizzolatti, "Motor facilitation during action observation: a magnetic stimulation study," Journal of Neurophysiology, vol. 73, no. 6, pp. 26082611, 1995.

[12] G. Buccino, S. Vogt, A. Ritzl et al., "Neural circuits underlying imitation learning of hand actions: an event-related fMRI study," Neuron, vol. 42, no. 2, pp. 323-334, 2004.

[13] S. Frenkel-Toledo, D. G. Liebermann, S. Bentin, and N. Soroker, "Dysfunction of the human mirror neuron system in ideomotor apraxia: evidence from mu suppression," Journal of Cognitive Neuroscience, vol. 28, no. 6, pp. 775-791, 2016.

[14] W. Chen, Q. Ye, X. Ji et al., "Mirror neuron system based therapy for aphasia rehabilitation," Frontiers in Psychology, vol. 6, p. 1665, 2015.
[15] T. F. Yuan and R. Hoff, "Mirror neuron system based therapy for emotional disorders," Medical Hypotheses, vol. 71, no. 5, pp. 722-726, 2008.

[16] A. Perry, S. N. Saunders, J. Stiso et al., "Effects of prefrontal cortex damage on emotion understanding: EEG and behavioural evidence," Brain, vol. 140, no. 4, pp. 1086-1099, 2017.

[17] K. A. Garrison, C. J. Winstein, and L. Aziz-Zadeh, “The mirror neuron system: a neural substrate for methods in stroke rehabilitation," Neurorehabilitation and Neural Repair, vol. 24, no. 5, pp. 404-412, 2010.

[18] J. A. Pineda, "Sensorimotor cortex as a critical component of an 'extended' mirror neuron system: does it solve the development, correspondence, and control problems in mirroring?," Behavioral and Brain Functions, vol. 4, no. 1, p. 47, 2008.

[19] G. Rizzolatti and C. Sinigaglia, "The functional role of the parieto-frontal mirror circuit: interpretations and misinterpretations," Nature Reviews Neuroscience, vol. 11, no. 4, pp. 264274, 2010.

[20] T. F. Yuan, W. Chen, C. Shan et al., "Activity-dependent neurorehabilitation beyond physical trainings: "mental exercise" through mirror neuron activation," CNS \& Neurological Disorders - Drug Targets, vol. 14, no. 10, pp. 1267-1271, 2015.

[21] E. Sarasso, M. Gemma, F. Agosta, M. Filippi, and R. Gatti, "Action observation training to improve motor function recovery: a systematic review," Archives of Physiotherapy, vol. 5, no. 1, p. 14, 2015.

[22] D. Ertelt, S. Small, A. Solodkin et al., "Action observation has a positive impact on rehabilitation of motor deficits after stroke," NeuroImage, vol. 36, pp. T164-T173, 2007.

[23] E. L. Altschuler, S. B. Wisdom, L. Stone et al., "Rehabilitation of hemiparesis after stroke with a mirror," The Lancet, vol. 353, no. 9169, pp. 2035-2036, 1999.

[24] K. Sugg, S. Müller, C. Winstein, D. Hathorn, and A. Dempsey, "Does action observation training with immediate physical practice improve hemiparetic upper-limb function in chronic stroke?," Neurorehabilitation and Neural Repair, vol. 29, no. 9, pp. 807-817, 2015.

[25] M. Franceschini, M. G. Ceravolo, M. Agosti et al., "Clinical relevance of action observation in upper-limb stroke rehabilitation: a possible role in recovery of functional dexterity. A randomized clinical trial," Neurorehabilitation and Neural Repair, vol. 26, no. 5, pp. 456-462, 2012.

[26] T. Cowles, A. Clark, K. Mares, G. Peryer, R. Stuck, and V. Pomeroy, "Observation-to-imitate plus practice could add little to physical therapy benefits within 31 days of stroke: translational randomized controlled trial," Neurorehabilitation and Neural Repair, vol. 27, no. 2, pp. 173-182, 2013.

[27] J. Fu, M. Zeng, F. Shen et al., "Effects of action observation therapy on upper extremity function, daily activities and motion evoked potential in cerebral infarction patients," Medicine, vol. 96, no. 42, article e8080, 2017.

[28] Y. Sun, W. Wei, Z. Luo, H. Gan, and X. Hu, "Improving motor imagery practice with synchronous action observation in stroke patients," Topics in Stroke Rehabilitation, vol. 23, no. 4, pp. 245-253, 2016.

[29] S. Caspers, K. Zilles, A. R. Laird, and S. B. Eickhoff, “ALE metaanalysis of action observation and imitation in the human brain," NeuroImage, vol. 50, no. 3, pp. 1148-1167, 2010.

[30] V. M. Pomeroy, C. A. Clark, J. S. G. Miller, J. C. Baron, H. S. Markus, and R. C. Tallis, "The potential for utilizing the "mirror neurone system" to enhance recovery of the severely 
affected upper limb early after stroke: a review and hypothesis," Neurorehabilitation and Neural Repair, vol. 19, no. 1, pp. 4-13, 2005.

[31] V. S. Ramachandran and E. L. Altschuler, "The use of visual feedback, in particular mirror visual feedback, in restoring brain function," Brain, vol. 132, no. 7, pp. 1693-1710, 2009.

[32] K. Eng, E. Siekierka, P. Pyk et al., "Interactive visuo-motor therapy system for stroke rehabilitation," Medical \& Biological Engineering \& Computing, vol. 45, no. 9, pp. 901-907, 2007.

[33] S. F. M. Toh and K. N. K. Fong, "Systematic review on the effectiveness of mirror therapy in training upper limb hemiparesis after stroke," Hong Kong Journal of Occupational Therapy, vol. 22, no. 2, pp. 84-95, 2012.

[34] S. Saleh, M. Yarossi, T. Manuweera, S. Adamovich, and E. Tunik, "Network interactions underlying mirror feedback in stroke: a dynamic causal modeling study," NeuroImage: Clinical, vol. 13, pp. 46-54, 2017.

[35] F. J. A. Deconinck, A. R. P. Smorenburg, A. Benham, A. Ledebt, M. G. Feltham, and G. J. P. Savelsbergh, "Reflections on mirror therapy: a systematic review of the effect of mirror visual feedback on the brain," Neurorehabilitation and Neural Repair, vol. 29, no. 4, pp. 349-361, 2015.

[36] A. M. Auriat, J. L. Neva, S. Peters, J. K. Ferris, and L. A. Boyd, "A review of transcranial magnetic stimulation and multimodal neuroimaging to characterize post-stroke neuroplasticity," Frontiers in Neurology, vol. 6, p. 226, 2015.

[37] H. M. Hobson and D. V. M. Bishop, "Mu suppression - a good measure of the human mirror neuron system?," Cortex, vol. 82, pp. 290-310, 2016.

[38] S. D. Muthukumaraswamy and K. D. Singh, "Modulation of the human mirror neuron system during cognitive activity," Psychophysiology, vol. 45, no. 6, pp. 896-905, 2008.

[39] R. S. Calabrò, A. Naro, M. Russo et al., "The role of virtual reality in improving motor performance as revealed by EEG: a randomized clinical trial," Journal of NeuroEngineering and Rehabilitation, vol. 14, no. 1, p. 53, 2017.

[40] F. Guo, Q. Xu, H. M. Abo Salem, Y. Yao, J. Lou, and X. Huang, "The neuronal correlates of mirror therapy: a functional magnetic resonance imaging study on mirror-induced visual illusions of ankle movements," Brain Research, vol. 1639, pp. 186-193, 2016.

[41] J. Kim, B. Lee, H. S. Lee, K. H. Shin, M. J. Kim, and E. Son, "Differences in brain waves of normal persons and stroke patients during action observation and motor imagery," Journal of Physical Therapy Science, vol. 26, no. 2, pp. 215-218, 2014.

[42] J. H. Kim and B. H. Lee, "Action observation training for functional activities after stroke: a pilot randomized controlled trial," NeuroRehabilitation, vol. 33, no. 4, pp. 565-574, 2013.

[43] M. Y. Chang, H. H. Kim, K. M. Kim, J. S. Oh, C. Jang, and T. H. Yoon, "Effects of observation of hand movements reflected in a mirror on cortical activation in patients with stroke," Journal of Physical Therapy Science, vol. 29, no. 1, pp. 38-42, 2017.

[44] M. Martin, K. Nitschke, L. Beume et al., "Brain activity underlying tool-related and imitative skills after major left hemisphere stroke," Brain, vol. 139, no. 5, pp. 1497-1516, 2016.

[45] S. H. Bae, W. S. Jeong, and K. Y. Kim, "Effects of mirror therapy on subacute stroke patients' brain waves and upper extremity functions," Journal of Physical Therapy Science, vol. 24, no. 11, pp. 1119-1122, 2012.
[46] A. Bhasin, R. Bhatia, S. S. Kumaran, S. Mohanty, and M. V. Padma Srivastava, "Neural interface of mirror therapy in chronic stroke patients: a functional magnetic resonance imaging study," Neurology India, vol. 60, no. 6, pp. 570-576, 2012.

[47] I. C. Brunner, J. S. Skouen, L. Ersland, and R. Gruner, "Plasticity and response to action observation: a longitudinal fMRI study of potential mirror neurons in patients with subacute stroke," Neurorehabilitation and Neural Repair, vol. 28, no. 9, pp. 874-884, 2014.

[48] C. Dettmers, V. Nedelko, and M. Ariel Schoenfeld, "Impact of left versus right hemisphere subcortical stroke on the neural processing of action observation and imagery," Restorative Neurology and Neuroscience, vol. 33, no. 5, pp. 701-712, 2015.

[49] S. Frenkel-Toledo, S. Bentin, A. Perry, D. G. Liebermann, and N. Soroker, "Mirror-neuron system recruitment by action observation: effects of focal brain damage on mu suppression," NeuroImage, vol. 87, pp. 127-137, 2014.

[50] K. A. Garrison, L. Aziz-Zadeh, S. W. Wong, S. L. Liew, and C. J. Winstein, "Modulating the motor system by action observation after stroke," Stroke, vol. 44, no. 8, pp. 2247-2253, 2013.

[51] E. J. Kuk, J. M. Kim, D. W. Oh, and H. J. Hwang, "Effects of action observation therapy on hand dexterity and EEG-based cortical activation patterns in patients with post-stroke hemiparesis," Topics in Stroke Rehabilitation, vol. 23, no. 5, pp. 318-325, 2016.

[52] M. E. Michielsen, R. W. Selles, J. N. van der Geest et al., "Motor recovery and cortical reorganization after mirror therapy in chronic stroke patients: a phase II randomized controlled trial," Neurorehabilitation and Neural Repair, vol. 25, no. 3, pp. 223-233, 2011.

[53] M. E. Michielsen, M. Smits, G. M. Ribbers et al., "The neuronal correlates of mirror therapy: an fMRI study on mirror induced visual illusions in patients with stroke," Journal of Neurology, Neurosurgery \& Psychiatry, vol. 82, no. 4, pp. 393-398, 2011.

[54] H. E. Rossiter, M. R. Borrelli, R. J. Borchert, D. Bradbury, and N. S. Ward, "Cortical mechanisms of mirror therapy after stroke," Neurorehabilitation and Neural Repair, vol. 29, no. 5, pp. 444-452, 2015.

[55] S. Saleh, S. V. Adamovich, and E. Tunik, "Mirrored feedback in chronic stroke: recruitment and effective connectivity of ipsilesional sensorimotor networks," Neurorehabilitation and Neural Repair, vol. 28, no. 4, pp. 344-354, 2014.

[56] A. J. Szameitat, S. Shen, A. Conforto, and A. Sterr, "Cortical activation during executed, imagined, observed, and passive wrist movements in healthy volunteers and stroke patients," NeuroImage, vol. 62, no. 1, pp. 266-280, 2012.

[57] J. Wang, C. Fritzsch, J. Bernarding et al., "Cerebral activation evoked by the mirror illusion of the hand in stroke patients compared to normal subjects," NeuroRehabilitation, vol. 33, no. 4, pp. 593-603, 2013.

[58] M. Tani, Y. Ono, M. Matsubara et al., "Action observation facilitates motor cortical activity in patients with stroke and hemiplegia," Neuroscience Research, 2017.

[59] M. Brunetti, N. Morkisch, C. Fritzsch et al., "Potential determinants of efficacy of mirror therapy in stroke patients - a pilot study," Restorative Neurology and Neuroscience, vol. 33, no. 4, pp. 421-434, 2015.

[60] P. Marque, D. Gasq, E. Castel-Lacanal, X. De Boissezon, and I. Loubinoux, "Post-stroke hemiplegia rehabilitation: evolution of the concepts," Annals of Physical and Rehabilitation Medicine, vol. 57, no. 8, pp. 520-529, 2014. 
[61] T. Askim, B. Indredavik, T. Vangberg, and A. Haberg, "Motor network changes associated with successful motor skill relearning after acute ischemic stroke: a longitudinal functional magnetic resonance imaging study," Neurorehabilitation and Neural Repair, vol. 23, no. 3, pp. 295-304, 2009.

[62] D. A. Nowak, C. Grefkes, M. Ameli, and G. R. Fink, "Interhemispheric competition after stroke: brain stimulation to enhance recovery of function of the affected hand," Neurorehabilitation and Neural Repair, vol. 23, no. 7, pp. 641-656, 2009.

[63] S. L. Small, G. Buccino, and A. Solodkin, "Brain repair after stroke-a novel neurological model," Nature Reviews Neurology, vol. 9, no. 12, pp. 698-707, 2013.

[64] L. Koski, A. Wohlschläger, H. Bekkering et al., "Modulation of motor and premotor activity during imitation of targetdirected actions," Cerebral Cortex, vol. 12, no. 8, pp. 847$855,2002$.

[65] S. L. Liew, K. A. Garrison, J. Werner, and L. Aziz-Zadeh, "The mirror neuron system: innovations and implications for occupational therapy," OTJR: Occupation, Participation and Health, vol. 32, no. 3, pp. 79-86, 2012.

[66] R. Newman-Norlund, H. T. van Schie, M. E. C. van Hoek, R. H. Cuijpers, and H. Bekkering, "The role of inferior frontal and parietal areas in differentiating meaningful and meaningless object-directed actions," Brain Research, vol. 1315, pp. 63$74,2010$.

[67] F. Hamzei, C. H. Lappchen, V. Glauche, I. Mader, M. Rijntjes, and C. Weiller, "Functional plasticity induced by mirror training: the mirror as the element connecting both hands to one hemisphere," Neurorehabilitation and Neural Repair, vol. 26, no. 5, pp. 484-496, 2012.

[68] Y. Kang, H. Park, H. Kim et al., "Upper extremity rehabilitation of stroke: facilitation of corticospinal excitability using virtual mirror paradigm," Journal of NeuroEngineering and Rehabilitation, vol. 9, no. 1, p. 71, 2012.

[69] T. Y. Shih, C. Y. Wu, K. C. Lin et al., "Effects of action observation therapy and mirror therapy after stroke on rehabilitation outcomes and neural mechanisms by MEG: study protocol for a randomized controlled trial," Trials, vol. 18, no. 1, p. 459, 2017.

[70] L. Fogassi, P. F. Ferrari, B. Gesierich, S. Rozzi, F. Chersi, and G. Rizzolatti, "Parietal lobe: from action organization to intention understanding," Science, vol. 308, no. 5722, pp. 662-667, 2005.

[71] S. Giaquinto, A. Cobianchi, F. Macera, and G. Nolfe, "EEG recordings in the course of recovery from stroke," Stroke, vol. 25, no. 11, pp. 2204-2209, 1994.

[72] C. Neuper, M. Wortz, and G. Pfurtscheller, "ERD/ERS patterns reflecting sensorimotor activation and deactivation," Progress in Brain Research, vol. 159, pp. 211-222, 2006.

[73] N. A. Fox, M. J. Bakermans-Kranenburg, K. H. Yoo et al., "Assessing human mirror activity with EEG mu rhythm: a meta-analysis," Psychological Bulletin, vol. 142, no. 3, pp. 291-313, 2016.

[74] H. M. Hobson and D. V. M. Bishop, "The interpretation of mu suppression as an index of mirror neuron activity: past, present and future," Royal Society Open Science, vol. 4, no. 3, article 160662, 2017.
[75] G. Rizzolatti, L. Cattaneo, M. Fabbri-Destro, and S. Rozzi, "Cortical mechanisms underlying the organization of goaldirected actions and mirror neuron-based action understanding," Physiological Reviews, vol. 94, no. 2, pp. 655-706, 2014.

[76] C. Babiloni, C. del Percio, F. Vecchio et al., "Alpha, beta and gamma electrocorticographic rhythms in somatosensory, motor, premotor and prefrontal cortical areas differ in movement execution and observation in humans," Clinical Neurophysiology, vol. 127, no. 1, pp. 641-654, 2016. 


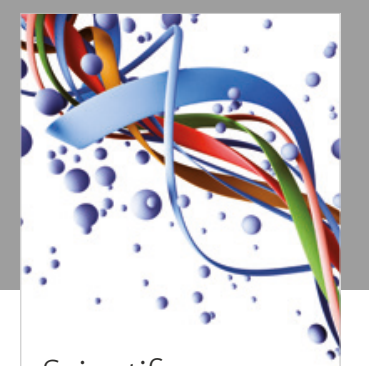

Scientifica
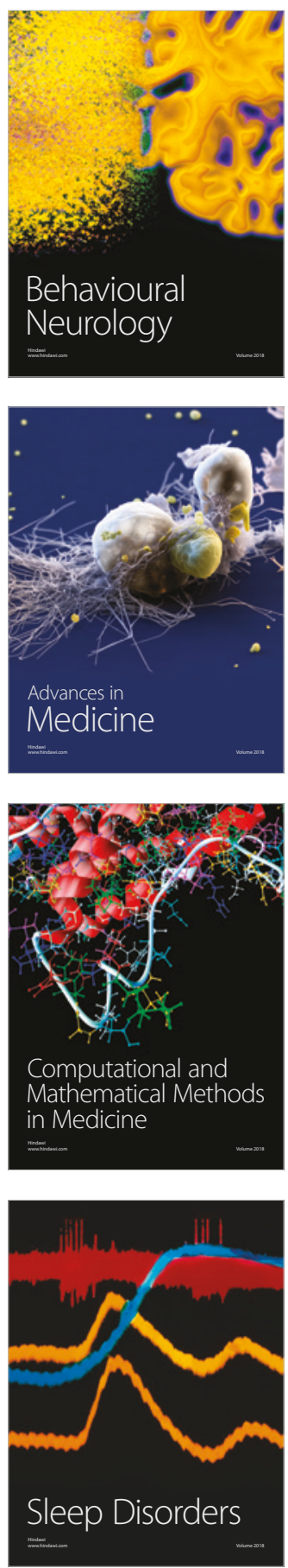

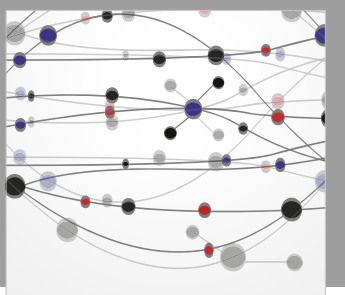

The Scientific World Journal

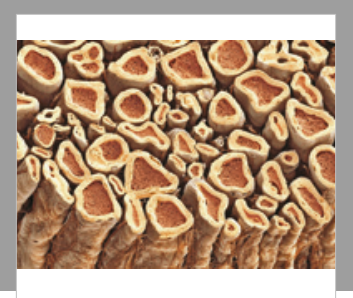

Case Reports in

Neurological Medicine

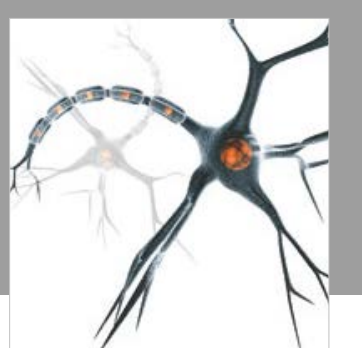

Neural Plasticity

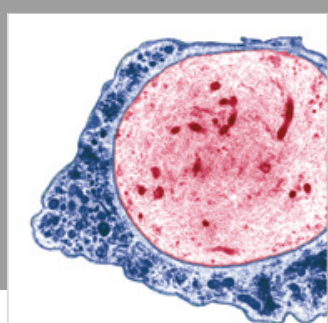

Multiple Sclerosis

International

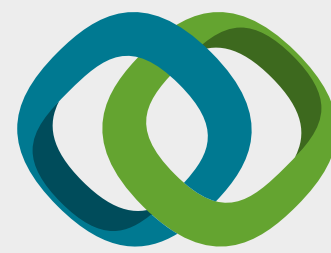

Hindawi

Submit your manuscripts at

www.hindawi.com
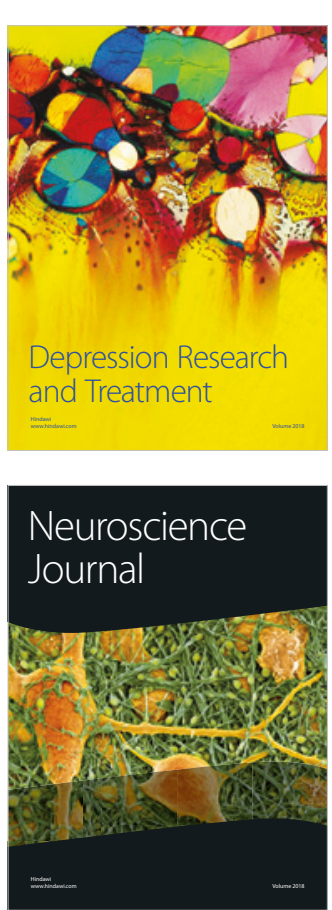

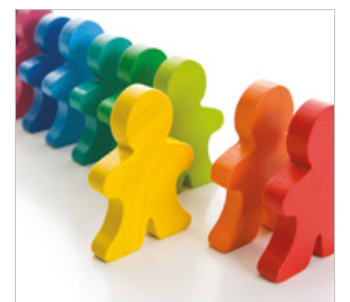

Autism

Research and Treatment
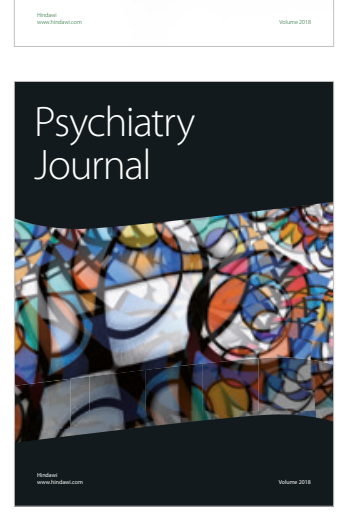
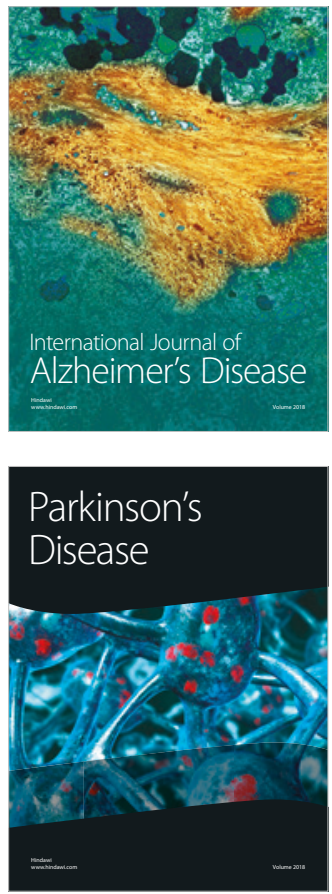
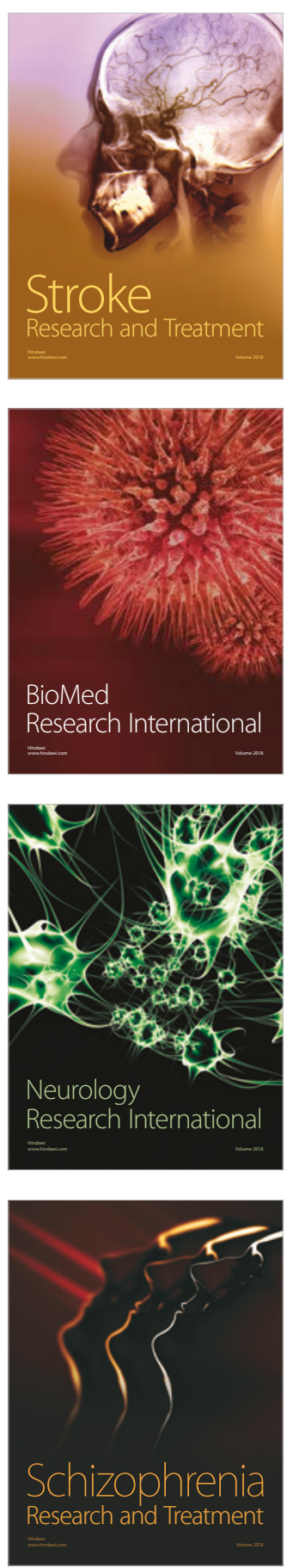